\title{
THERMODYNAMICS OF CARRIER DISTRIBUTION WITHIN LOCALIZED ELECTRONIC STATES WITH A BROAD GAUSSIAN ENERGY DISTRIBUTION AND ITS EFFECT ON LUMINESCENCE BEHAVIOR OF LOCALIZED STATES
}

\author{
Q. $\mathrm{Li}^{1}$, S. J. Xu ${ }^{1 *}$, W. C. Cheng ${ }^{1}$, M. H. Xie ${ }^{1}$, S. Y. Tong ${ }^{1 \dagger}$ and H. Yang ${ }^{2}$ \\ ${ }^{1}$ Department of Physics and HKU-CAS Joint Laboratory on New Materials, \\ The University of Hong Kong, Pokfulam Road, Hong Kong, China. \\ ${ }^{2}$ State Key Laboratory of Integrated Optoelectronics, Institute of \\ Semiconductors, Chinese Academy of Sciences, 100083, Beijing, China.
}

\begin{abstract}
A new carrier distribution function is derived to describe the the thermodynamics of carriers within localized states with a broad energy distribution. With the aid of this function, several luminescence behaviors, i.e., (1) fast redshift of peak positions at low temperatures; (2) decrease of full-width-at-half-maximum (FWHM) of luminescence band at low temperatures; and (3) blueshift of peak positions at high temperatures, frequently observed in above material systems can be explained. Finally, the model is employed to fit the experimental data of cubic InGaN thin film grown on GaAs. A good agreement between the experimental data and the theoretical fitting is obtained.
\end{abstract}

\section{INTRODUCTION}

Presence of localized electronic states with a broad energy distribution is a common characteristic feature of many material systems such as self-assembled quantum dots, GaInP and InGaN alloys. Generally, the random alloy disorder exists in ternary semiconductor materials. However, in InGaN, there have been more and more evidences [1-4] showing that instead of a simple random statistical distribution of In and Ga atoms, In-rich clusters with a broad size distribution from the atomic scale up to $100 \mathrm{~nm}$ naturally form. These In-rich clusters create a complex spatial landscape for the electronic states and leads to the presence of localized states with energies of several hundred meV below the bandgap [5]. Several anomalous phenomena such as the coexistence of high luminescence efficiency and high density of threading dislocations as well as the blue-shift of the luminescence peak position with increasing temperature, etc., are quite often observed in InGaN alloys grown on different substrates with different growth techniques. A band-tail state emission model has been proposed by Eliseev $e t$ al. [6] to interpret the blue-shift of the emission peak with temperature. Another interesting example is In(Ga)As/GaAs self-assembled quantum dots (QDs). The Gaussian-like size distribution of $\operatorname{In}(\mathrm{Ga}) \mathrm{As} / \mathrm{GaAs}$ QDs [7] results in localized electronic states with a Gaussian energy distribution. Fast red-shift of peak position of luminescence band at low-temperature region in such materials was observed [8].

In the present work, a temperature-dependent distribution function taking into account thermal activation, transfer and re-capture of localized carriers is derived to describe the thermodynamics of carriers within localized states. The energetic peak positions and full-width-at-half-maximum (FWHM) of the distribution function are

\footnotetext{
${ }^{*}$ Corresponding author. Address: Department of Physics, University of Hong Kong, Pokfulam Road, Hong Kong SAR. Phone: (852) 22415636. Fax: (852) 25599152. Email: sjxu@hkucc.hku.hk.

${ }^{\dagger}$ Present address: Department of Physics and Materials Science, City University of Hong Kong, 83 Tat Chee Avenue, Kowloon, Hong Kong, China.
} 
calculated as a function of temperature. It is found that the temperature behavior of photoluminescence (PL) spectra in the cubic InGaN film can be interpreted very well with the model.

\section{THEORY AND EXPERIMENTAL}

\subsection{Distribution function}

In order to explain the fast red-shift of luminescence band of self-assembled InAs/GaAs QDs, a "coupled" InAs QDs model had been proposed by $\mathrm{Xu}$ et al. [8]. In the model, carriers are thermally activated away from localized electronic states such as InAs QDs, transfer and be re-captured by other localized states. Under weak excitation condition, the dynamics of the carriers can be described by the following rate equation [9]:

$$
\begin{aligned}
\frac{d N(E, T)}{d t}= & G(E)+\alpha \int_{E^{\prime}} \frac{N\left(E^{\prime}, T\right)}{\tau_{t r}} e^{\left(E^{\prime}-E_{a}\right) / k_{B} T} d E^{\prime} \frac{\rho(E)}{\int_{E} \rho(E) d E} \\
& -\frac{N(E, T)}{\tau_{t r}} e^{\left(E-E_{a}\right) / k_{B} T}-\frac{N(E, T)}{\tau_{r}}
\end{aligned}
$$

where the energy distribution of the localized states is assumed to be a Gaussian-like function, that is, $\rho(E) \propto$ $\exp \left[-\left(E-E_{0}\right) / 2 \sigma^{2}\right]$, where $E_{0}$ and $\sigma$ represent the center and the width of of the distribution respectively. $N(E, T)$ is the distribution of the localized excitons, $G(E)$ is the generation rate which is proportional to the distribution of the localized states, $E_{a}$ represents a barrier level that the carriers must overcome to transfer, $k_{B}$ is the Boltzmann constant, $T$ is the lattice temperature, $\tau_{t r}$ is the carrier transfer time, $\tau_{r}$ is the carrier recombination time, and $\alpha$ represents the carrier re-capture coefficient. The second term on the right side of Eq. (1) describes that the re-captured process of the thermal-activated carriers by the localized states, the third term describes the thermal-activation of carriers from the localized states and the last term describes the carriers recombination. Under steady-state condition, that is, $d N(E, T) / d t=0$, the solution of Eq. (1) can be written as

$$
N(E, T) \propto \frac{e^{-\left(E-E_{0}\right)^{2} / 2 \sigma^{2}}}{e^{\left(E-E_{a}\right) / k_{B} T}+\tau_{t r} / \tau_{r}} .
$$

The above is the temperature-dependent distribution function describes the thermodynamics of carriers within the electronic localized states.

If all localized excitons are assumed to have same radiative recombination rate and carrier transfer time, Eq. (2) can be used to represent the shape of the luminescence band. In the following the distribution function is used to explain several luminescence behavior observed in our InGaN sample.

\subsection{Anomalous temperature dependence of peak positions of InGaN.}

The $\mathrm{In}_{x} \mathrm{Ga}_{1-x} \mathrm{~N}$ epilayers investigated here were grown on $\mathrm{GaAs}(001)$ substrates with metal-organic vapor phase epitaxy. Prior to the growth of about $0.3 \mu \mathrm{m} \mathrm{InGaN} \mathrm{layers,} \mathrm{an} \mathrm{about} 0.6 \mu \mathrm{m}$ thick zinc-blende GaN buffer layer was deposited. Further details of the growth can be found elsewhere [10]. The In concentration of the sample investigated here is $21 \%$ [10]. The PL spectra of the sample has been previously reported [11].

Temperature dependence of the peak positions (solid circles) of the luminescence band of the sample is shown in Fig. 1(A). Obviously, it does not follow the behavior of a semiconductor band gap predicted by either Varshni [12] or Bose-Einstein [13] formula. When the temperature is below $160 \mathrm{~K}$, the luminescence band red-shifts faster than that predicted by Varshni's empirical formula as the temperature increases. However, a blue-shift of the luminescence peak takes place when the temperature is above $160 \mathrm{~K}$. The temperature-induced blue-shift of the luminescence peak has been observed in a lot of different semiconductor materials $[14,15]$ including InGaN $[6]$.

Let $\partial N(E, T) / \partial E=0$ in Eq. (2), the peak positions of $N(E, T)$ at different temperatures can be derived. We find that when

$$
E=E_{0}-x(T) \cdot k_{B} T,
$$

$N(E, T)$ reaches its maximum values. The temperature-dependent dimensionless coefficient $x(T)$ in Eq. (3) can be obtained by numerically solving following equation

$$
x e^{x}=\left(\frac{\tau_{r}}{\tau_{t r}}\right)\left[\left(\frac{\sigma}{k_{B} T}\right)^{2}-x\right] e^{\left(E_{0}-E_{a}\right) / k_{B} T} .
$$



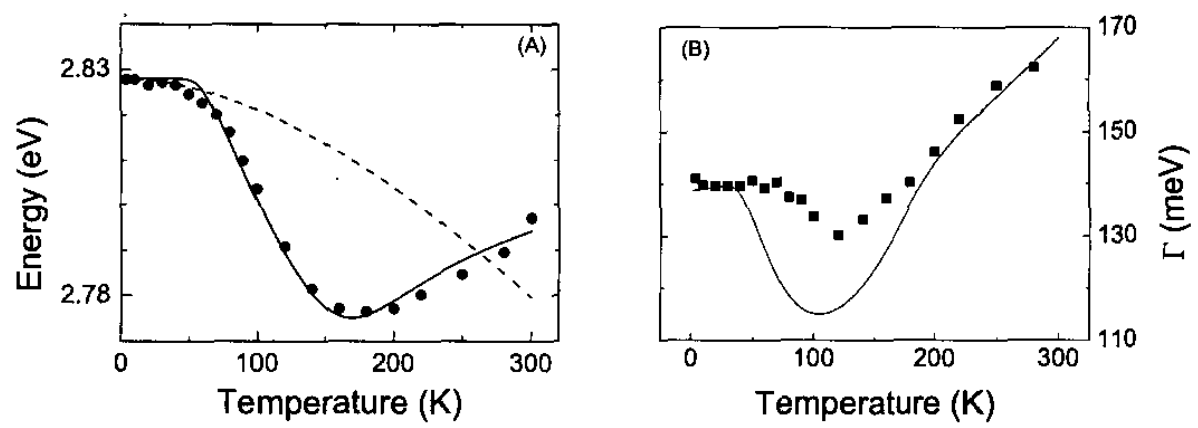

Figure 1: (A) Peak energies of the luminescence band of cubic InGaN as a function of temperature. The solid circles are the experimental data, the dashed line is calculated from Varshni's formula $\left(\alpha=0.5 \times 10^{-3} \mathrm{eV} / \mathrm{K}\right.$, $\Theta=630 \mathrm{~K}$ taken from Ref. [6]) and the solid line is the fitting curve according to Eqs. (3) and (4). (B) Linewidth of luminescence peak of the sample as a function of the temperature (solid squares). The solid line is the calculated FWHM taking into account both the contribution from phonon scattering and the contribution from the carriers' thermal re-distribution.

Eq. (4) has only one solution at $0<x<\left(\sigma / k_{B} T\right)^{2}$.

Eqs. (3) and (4) are employed to fit the energetic peak positions of PL spectra of the sample. The solid line in Fig. 1(A) is the fitting result to the experimental data of the sample. The fitting parameters are $E_{0}=2.828$ $\mathrm{eV}, E_{a}-E_{0}=75 \mathrm{meV}, \tau_{t r} / \tau_{r}=0.022 \mathrm{ps} / 800 \mathrm{ps}, \sigma=30 \mathrm{meV}$. Quite good agreement between the theory and the experiment is obtained. The energy difference $E_{a}-E_{0}$ reflects the magnitude of the carrier localization. Our values of $E_{a}-E_{0}$ are in good agreement with the localization depth in the wurtzite $\operatorname{In}_{0.15} \mathrm{Ga}_{0.85} \mathrm{~N}$ reported by Yang et al. [16]. As has been previousily pointed out [11], the present model can be approximated to Eliseev's band-tail model [6] at high temperature region.

\subsection{Shrinkage of FWHM at low-temperature region}

The linewidth of the PL peak is $\sim 140 \mathrm{meV}$ at low temperature. When $T<60 \mathrm{~K}$, the peak linewidth almost remains unchanged. When $T>60 \mathrm{~K}$, the peak linewidth starts to shrink. The peak width decreases to $\sim 130 \mathrm{meV}$ at 120 $\mathrm{K}$. When the temperature is above $120 \mathrm{~K}$, the peak width increases. The linewidth of the peak is depicted as a function of temperature in Fig. 1(B). The phenomenon of linewidth shrinkage in the low-temperature region was also observed in InAs/GaAs QDs [8]. The solid line is the calculated linewidth. There are two contributions to the PL peak linewidth. One is from the carriers' thermal re-distribution within localized states and the other stems from intrinsic phonon scattering, that is, $\Gamma_{\text {total }}(T)=\Gamma_{c}(T)+\Gamma_{p h}(T)$. The linewidth change caused by the carriers' thermal re-distribution, $\Gamma_{c}(T)$, can be numerically calculated directly from Eq. (2). The peak linewidth broadening due to phonon scattering can be given by $\Gamma_{p h}(T)=\Gamma_{0}+\sigma T+\gamma\left[\exp \left(\hbar \omega_{L O} / k_{B} T\right)-1\right]^{-1}[17]$. Where $\Gamma_{0}$ is a constant, standing for the impurity and imperfection scattering. The second term arises from the scattering of the excitons by acoustic phonons, exhibiting the linear temperature dependence. $\sigma$ is the exciton-acoustic phonon coupling strength. The last term is due to the interaction of the excitons with the LO phonons. Parameters $\Gamma_{0}=69$ $\mathrm{meV}, \sigma=0.036 \mathrm{meV} / \mathrm{K}, \gamma=400 \mathrm{meV}$ and $\hbar \omega_{L O}=80 \mathrm{meV}$ are adopted [18] to obtain a reasonably good agreement between the calculation (solid line) and the experimental data (solid squares) (as shown in Fig. 1(B)). It is clear that the thermal re-distribution of carriers within localized states results in the shrinkage of PL peak linewidth at low temperature region.

\section{CONCLUSION}

A new carrier distribution function describing thermodynamics of carriers in localized states is developed. Utilizing the function, several anomalous luminescence behavior of the InGaN alloys are explained. It is expected that the model can be expanded to other materials within which localized electronic states distribute over broad energy range. 


\section{ACKNOWLEDGMENTS}

The work was supported by HKU URC Grant (No. 10203533) and partly supported by the AOE program of lightemitting materials at HKU and NSFC/RGC Joint Research Scheme (N_HKU028/00) and the Croucher Foundation of Hong Kong.

\section{References}

[1] K. P. O’Donnell, R. W. Martin, and P. G. Middleton, Phys. Rev. Lett. 82, 237 (1999).

[2] S. F. Chichibu, K. Wada, J. Müllhäuser, O. Brandt, K. H. Ploog, T. Mizutani, A. Setoguchi, R. Nakai, M. Sugiyama, H. Nakanishi, K. Torii, T. Deguchi, T. Sota, and S. Nakamura, Appl. Phys. Lett. 76, 1671 (2000).

[3] V. Lemos, E. Silveira, J. R. Leite, A. Tabata, R. Trentin, L. M. R. Scolfaro, T. Frey, D. J. As, D. Schikora, and K. Lischka, Phys. Rev. Lett. 84, 3666 (2000).

[4] H. Chen, R. M. Feenstra, J. E. Northrup, T. Zywietz, and J. Neugebauer, Phys. Rev. Lett. 85, 1902 (2000).

[5] N. M. Johnson, A. V. Nurmikko, and S. P. DenBaars, Phys. Today 53, 31 (2000).

[6] P. G. Eliseev, P. Perlin, J. Lee, and M. Osiński, Appl. Phys. Lett. 71, 569 (1997).

[7] Y. Ebiko, S. Muto, D. Suzuki, S. Itoh, K. Shiramine, and T. Haga, Phys. Rev. Lett. 80, 2650 (1998).

[8] Z. Y. Xu, Z. D. Lu, X. P. Yang, Z. L. Yuan, B. Z. Zheng, J. Z. Xu, W. K. Ge, Y. Wang, J. Wang, and L. L. Chang, Phys. Rev. B 54, 11528 (1996).

[9] Z. Y. Xu, Z. D. Lu, Z. L. Yuan, X. P. Yang, B. Z. Zheng, J. Z. Xu, W. K. Ge, Y. Wang, J. Wang, and L. L. Chang, Supperlattice Microst. 23, 381 (1998).

[10] J. B. Li, H. Yang, L. X. Zheng, D. P. Xu, and Y. T. Wang, MRS Internet J. Nitride Semicond. Res. 4S1, G3.25 (1999).

[11] Q. Li, S. J. Xu, W. C. Cheng, L. X. Zheng, M. H. Xie, S. Y. Tong, C. M. Che, and H. Yang, Appl. Phys. Lett. 79, $1810(2001)$.

[12] Y. P. Varshni, Physica 34, 149 (1967).

[13] L. Vina, S. Logothetidis, and M. Cardona, Phys. Rev. B 30, 1979 (1984).

[14] L. Bergman, M. Dutta, M. A. Stroscio, S. M. Komirenko, C. J. Eiting, D. J. H. Lambert, H. K. Kwon, and R. D. Dupuis, Appl. Phys. Lett. 76, 1969 (2000).

[15] S. Guha, Q. Cai, M. Chandrasekhar, H. R. Chandrasekhar, H. Kim, A. D. Alvarenga, R. Vogelgesang, A. K. Ramdas, and M. R. Melloch, Phys. Rev. B 58, 7222 (1998).

[16] H. C. Yang, T. Y. Lin, and Y. F. Chen, Appl. Phys. Lett. 78, 338 (2001).

[17] S. Rudin, T. L. Reinecke, and B. Segall, Phys. Rev. B 42, 11218 (1990).

[18] S. J. Xu, L. X. Zheng, S. H. Cheung, M. H. Xie, S. Y. Tong, and H. Yang, Appl. Phys. Lett. 81, 4389 (2002). 